\title{
Experimental study of source of background noise in muon radiography using emulsion film detectors
}

\author{
R. Nishiyama ${ }^{1}$, S. Miyamoto ${ }^{1}$, and N. Naganawa ${ }^{2}$ \\ ${ }^{1}$ Earthquake Research Institute, The University of Tokyo, Tokyo, Japan \\ ${ }^{2}$ Department of Physics, Graduate School of Science, Nagoya University, Nagoya, Japan \\ Correspondence to: R. Nishiyama (nisiyama@eri.u-tokyo.ac.jp) \\ Received: 13 November 2013 - Published in Geosci. Instrum. Method. Data Syst. Discuss.: 4 December 2013 \\ Revised: 22 March 2014 - Accepted: 28 March 2014 - Published: 16 April 2014
}

\begin{abstract}
The aim of this study is to ascertain and confirm the source of background noise in cosmic-ray muon radiography (muography) using emulsion film detectors. For this, we build two types of emulsion detectors with different momentum thresholds and perform test measurements of an actual geoscientific target. This experiment reveals that contamination of nonsignal particles with momenta of less than $2 \mathrm{GeV} \mathrm{c}^{-1}$ cause significant systematic errors for the density estimation of muography. Utilizing the results of precedent studies, we conclude that the origin of these low-momentum particles is either electromagnetic components of air showers or cosmic-ray muons scattered in topographic material. In this paper, we analyze the emulsion data in detail, including the film-inefficiency compensation and momentum selection by applying an upper bound to the chi-square distribution for the data.
\end{abstract}

\section{Introduction}

Muon radiography (muography) is a noninvasive inspection technique that exploits the high penetration power of cosmicray muons (CR muons). Use of this technique has spread to a variety of fields, such as volcanology (e.g., Tanaka et al., 2007; Lesparre et al., 2012; Portal et al., 2013), and hydrology (e.g., Tanaka et al., 2011b). The muography technique measures the absorption rate of cosmic-ray muons inside the target material. The energy spectrum of CR muons and their interaction with matter have been extensively investigated, and the attenuation of muon flux can be used to derive the amount of matter present along the muon trajectories. The amount of matter is given in units of density times length (density length, $100 \mathrm{~g} \mathrm{~cm}^{-2}=1 \mathrm{~m}$ water equivalent - w.e.).

Muography of mountains has been performed by placing a position-sensitive detector for minimum ionization particles (MIPs) at the foot of the target mountain. These measurements aim to detect muons that cross the detector nearly horizontally. Since the flux of horizontal muons is approximately one-tenth to one-hundredth of that of vertical muons and decreases drastically when passing through material, the number of signal muons is very small. Because of this small number, the background noise caused by nonsignal particles must be excluded.

A requirement of signal muons is that they must pass through the target material with a straight trajectory. These muons should have high momentum when they are detected. However, we assume that the following issues cause background particles: (1) electromagnetic components of air showers, and (2) CR muons deflected at large angles through the material. As discussed in the next section, most of these background particles have momentum of less than $2 \mathrm{GeV} \mathrm{c}^{-1}$. These low-momentum particles are scattered into random directions by the material around the detector. They may pass through the detector by chance and thus generate background noise.

Based on the above discussion, we conclude that a muography detector must have a momentum separation function to reduce systematic errors arising from low-momentum background particles. To validate this assumption, we built two types of detectors with different momentum thresholds using emulsion films, and we performed a test measurement of an actual geoscientific target. In this paper, we describe the analysis method of the emulsion film data. Compensation for 
the film inefficiency is described in detail, since this process is crucial for converting the number of signals into the particle flux. Using the particle fluxes from the two detectors, we check the existence of low-momentum background particles and we discuss their origin.

\section{Signal and noise in muography}

\subsection{Signal muons}

Muons are the most numerous charged particles at sea level (Beringer et al., 2012). They are produced high in the atmosphere by the decay of charged mesons (pions and kaons). Their energy and angular distribution reflect a convolution of their production spectrum, energy loss in the atmosphere and decay. The energy spectra of nearly horizontal muons were investigated in detail in the MUTRON (Matsuno et al., 1984) and DEIS (Allkofer et al., 1985) experiments in the 1980s. In this study, we use the analytic formula for describing the muon energy spectrum developed by Matsuno et al. (1984). Although the formula does not take into account the rigidity cutoff due to the geomagnetism of the Earth, or the variation of solar activity, these effects affect the muon flux only in the gigaelectronvolt range, and the variation is only $10 \%$. The mean momentum of vertical muons is $\sim 4 \mathrm{GeV} \mathrm{c}^{-1}$ and that of nearly horizontal muons is $\sim 10 \mathrm{GeV} \mathrm{c}^{-1}$. The background noise candidates, described in the next subsections, typically have lower momentum than the signal muons.

\subsection{Electromagnetic components of air-showers}

The electromagnetic components from electromagnetic cascades, which are initiated by the decay of neutral and charged mesons, can be sources of background noise in muography, since they are scattered into the detector from random directions. Electrons and positrons are most numerous near their critical energy ( $81 \mathrm{MeV}$ in air). The total vertical intensity of electrons plus positrons is roughly, 30, 6, and $0.2 \mathrm{~m}^{-2} \mathrm{~s}^{-1} \mathrm{sr}^{-1}$ above 10,100 , and $1000 \mathrm{MeV}$ respectively. A dedicated study (Golden et al., 1995) shows that the ratio of electrons plus positrons to muons is as much as onethird at a few hundreds of $\mathrm{MeV}$, and it decreases drastically to one-hundredth at $2 \mathrm{GeV}$. This precedent work implies that this electromagnetic component dominates the ionizing particles at ground level below the critical energy $(81 \mathrm{MeV})$. Unfortunately, there are no sufficient experimental data on the angular dependence of the energy spectrum.

\subsection{Scattered muons}

Some CR muons are deflected at large angles in a target material due to multiple coulomb scattering and bremsstrahlung. These muons can also be a source of background noise in muography, since they lose their original information of direction as they pass through the material.
This can be shown by performing a simple Monte Carlo simulation in which CR muons are injected into a certain thickness of material. We assumed a quartz-made (density $\left.=2200 \mathrm{~kg} \mathrm{~m}^{-3}\right), 100 \mathrm{~m}$-thick wall in a computational volume of the Geant 4 toolkit (Agostinelli et al., 2003), and injected 100000 muons perpendicular to the wall. The incident momenta were adjusted to the $\mathrm{CR}$ muon momentum spectrum above $10 \mathrm{GeV} \mathrm{c}^{-1}$ for a zenith angle of $80^{\circ}$. Of the injected muons, 23512 muons penetrated the wall and emerged from the other side. We extracted the momentum $p_{\text {out }}$ of the ejected muons and their deflection angles $\Delta \vartheta_{\text {out }}$ (Fig. 1). We found that $\Delta \vartheta_{\text {out }}$ has a long-tail distribution up to $0.3 \mathrm{rad}$, and that 628 muons ( $3 \%$ of the penetrated muons) were deflected with more than $0.1 \mathrm{rad}$. Out of these 628 muons, $568(90 \%)$ muons of were low-momentum $\left(p_{\text {out }}<2.0 \mathrm{GeV} \mathrm{c}^{-1}\right)$. Thus, these low-momentum muons could hit the detector from unexpected paths through the topography and cause a systematic error in the density estimation.

\section{Momentum filtering in emulsion cloud chamber}

\subsection{Nuclear emulsion detector}

Nuclear emulsion films are employed in three-dimensional, charged particle detectors that have submicron position resolution with no dead space and no dead time. The emulsion film consists of plastic base and nuclear emulsion gel, which covers the base on both sides. The main components of the nuclear emulsion gel are basically the same as ordinary photographic films, $\mathrm{AgBr}$ crystals and gelatin. The $\mathrm{AgBr}$ crystals in a nuclear emulsion gel are sensitive to charged particles (Nakamura et al., 2006). After the nuclear emulsions are developed, the trajectories of the charged particles are recorded as three-dimensional lines of the silver grains (Fig. 2). A typical size of the silver grain is about $0.6 \mu \mathrm{m}$. These lines of silver grains are identified by an automated track readout system (Morishima and Nakano, 2010; Arrabito et al., 2006).

For the application of muography, several types of detector have been used, such as a scintillation type (e.g., Tanaka et al., 2011a; Lesparre et al., 2012; Anastasio et al., 2013), a gas chamber type (e.g., Barnaföldi et al. 2012; Cârloganu et al., 2013), and an emulsion type (Tanaka et al., 2007). The greatest advantage of the emulsion detectors is that they are portable and do not require electricity to operate. In addition, emulsion films enable us to trace the particle trajectories with very high resolutions (position resolution: a few micrometers, angular resolution: a few milliradians).

\subsection{Momentum measurement with ECC}

When a charged particle passes through the material, the particle is scattered by the electric field of the nuclei (multiple coulomb scattering). The distribution of the scattering angle is well described as a Gaussian probability density function 

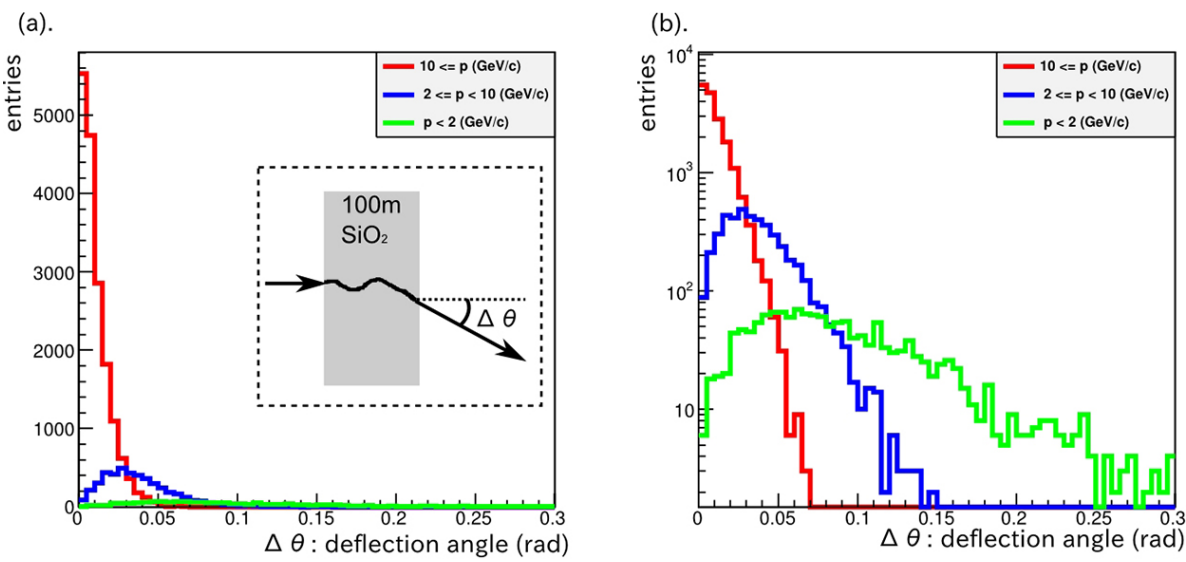

Fig. 1. Deflection angles of CR muons after passing through $100 \mathrm{~m}$ thickness of quartz ((a) linear scale, (b) logarithmic scale). The injection momenta are adjusted to the CR muon momentum spectrum for a zenith angle of $80^{\circ}$. The histogram is divided into three color parts based on the momenta of the ejected muons, green: $p_{\text {out }}<2 \mathrm{GeVc}^{-1}$, blue: $2 \mathrm{GeVc}^{-1} \leq p_{\text {out }}<10 \mathrm{GeVc}^{-1}$, and red: $10 \mathrm{GeVc}{ }^{-1}<p_{\text {out }}$.

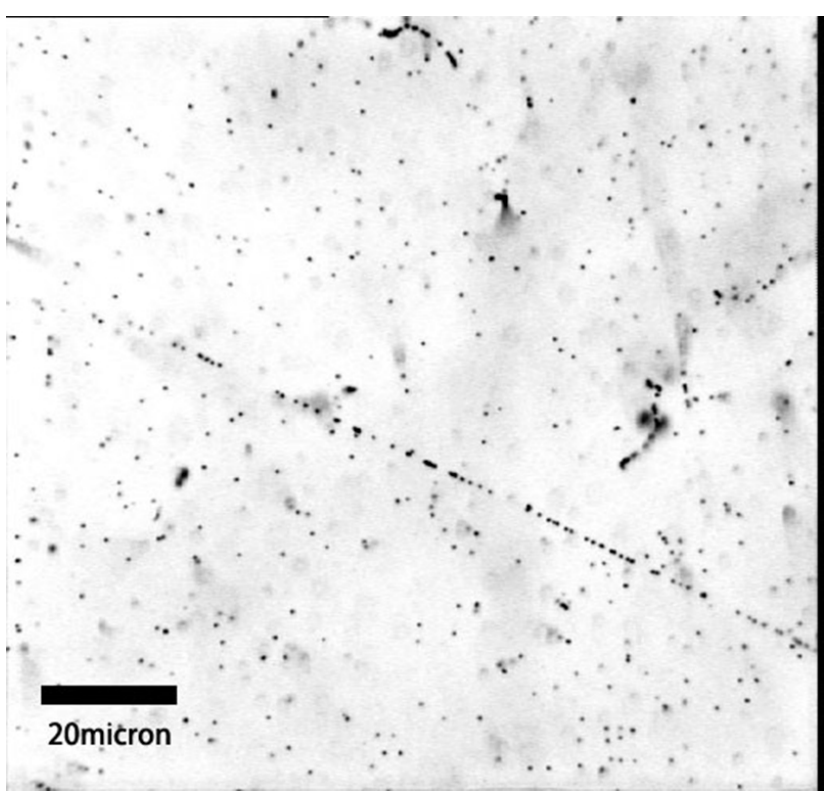

Fig. 2. Microscopic image of developed nuclear emulsion film.

whose standard deviation is given by

$\sigma_{\text {scatt }}=\frac{13.6 \mathrm{MeV} \mathrm{c}^{-1}}{p \beta} \sqrt{\frac{t}{X_{0}}}\left\{1+0.038 \log \left(\frac{t}{X_{0}}\right)\right\}$,

where $p$ is the momentum, $\beta$ the relative velocity to the speed of light, and $t / X_{0}$ the thickness of the scatterer in radiation length (Lynch and Dahl, 1991) This Gaussian approximation is valid for small deflection angles (less than a few $\sigma_{\text {scatt }}$ ).

The emulsion cloud chamber (ECC) is a modular structure made of a sandwich of passive material plates such as lead interleaved with emulsion film layers. Due to the very high position resolution of emulsion films, we can determine the momentum of the traversing particles by measuring small deflections of tracks caused by multiple coulomb scattering (De Serio et al., 2003). Since the typical angular resolution of tracks in an emulsion film is $5 \mathrm{mrad}$, we can identify scattering angles of $1 \mathrm{GeV} \mathrm{c}^{-1}$ particles in a $1 \mathrm{~mm}$-thick lead plate.

\section{Experimental setup and coordinate systems}

We prepared two types of detectors using OPERA (Oscillation Project with Emulsion-tRacking Apparatus) emulsion films to investigate the features of low-momentum noise particles. One is a stack of four emulsion films (quartet detector). The other is a stack of 20 emulsion films and nine $1 \mathrm{~mm}$-thick lead plates (ECC detector). Both detectors were sandwiched between two $3 \mathrm{~mm}$-thick stainless plates to protect them from ambient radioactive particles (Tanaka et al., 2007).

As discussed in Sect. 5.3.2, the momentum thresholds of these two detectors are 0.2 (quartet) and $2.0 \mathrm{GeV} \mathrm{c}^{-1}$ (ECC), respectively. Schematic cross views of these detectors are shown in Fig. 3. We installed the detectors $500 \mathrm{~m}$ west of the summit of Mt. Showa-Shinzan in the Usu volcanic region in Hokkaido, Japan. These detectors were placed in front of Mt. Showa-Shinzan. Behind the detectors was another larger volcano, Mt. Usu. One week before the installation, all the OPERA films were refreshed in a high temperature and humidity place, and were packed in plastic envelopes in a less humid place $(\sim 50 \%)$. The detectors were fabricated just before the installation to reduce the misreconstruction of the tracks recorded during transportation. The exposure time was 168 days (21 November 2011-7 May 2012). The maximum temperature was $15.9^{\circ} \mathrm{C}$ and the minimum was $-8.8^{\circ} \mathrm{C}$ around the detector site during the exposure (Japan Meteorological Agency).

We employed a Cartesian coordinate system with the $z$ axis normal to the detector plane (Fig. 3c) to identify the track position and direction. The track orientation is 

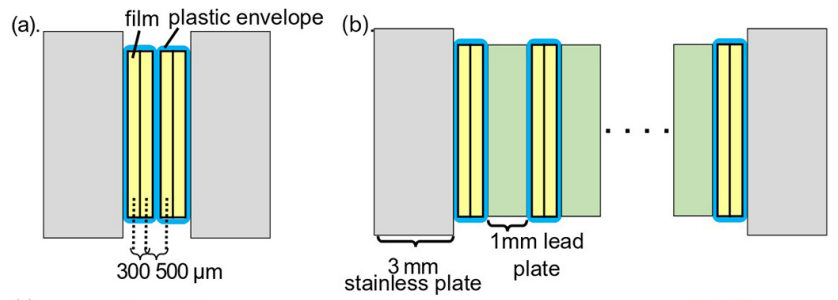

(c)
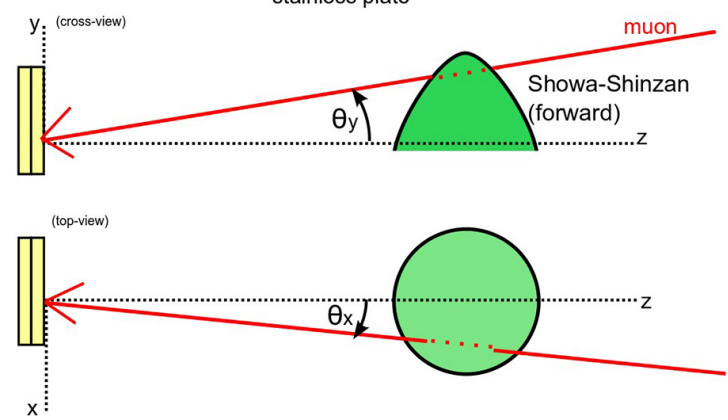

Fig. 3. Schematic cross views of two types of emulsion detectors. (a) Quartet detector: emulsion films, packed in twos in plastic light-shielding envelopes stacked between $3 \mathrm{~mm}$-thick stainless steel plates to ensure planarity. (b) ECC detector: ten envelopes and nine $1 \mathrm{~mm}$-thick lead plates interleaved between $3 \mathrm{~mm}$-thick stainless steel plates. (c) Emulsion coordinate system for identifying the position and direction of particles.

represented by $\tan \theta_{x}$ and $\tan \theta_{y}$, where $\theta_{x}$ and $\theta_{y}$ are the angles with respect to the film's normal projections on the $x z$ and $y z$ planes, respectively. The inclination angle $\tan \theta=$ $\sqrt{\tan ^{2} \theta_{x}+\tan ^{2} \theta_{y}}$, the angle between the track and the detector plane, is an important parameter since the angular resolution and the film efficiency depend strongly on $\theta$. While the ECC detector was placed vertically on the ground, we placed the quartet detector at a slant of $\alpha=11^{\circ}$ to the ground. Thus, in order to compare the results, the quartet coordinates $\left(\theta_{x}^{\mathrm{Q}}, \theta_{y}^{\mathrm{Q}}\right)$ have to be transformed to the ECC coordinates $\left(\theta_{x}, \theta_{y}\right)$ via the relation

$\tan \theta_{x}^{\mathrm{Q}}=\frac{\tan \theta_{x}}{\sin \alpha \tan \theta_{y}+\cos \alpha}$,

$\tan \theta_{y}^{\mathrm{Q}}=\frac{\cos \alpha \tan \theta_{y}-\sin \alpha}{\sin \alpha \tan \theta_{y}+\cos \alpha}$.

In this coordinate system, $\tan \theta_{y}>0$ indicates particles coming from the front, $\tan \theta_{y}<0$ particles coming from the rear, and $\tan \theta_{y}=0$ horizontal particles, because almost all the cosmic particles travel downward. The thickness of the rock along the radial direction from the detector is shown in Fig. 4.

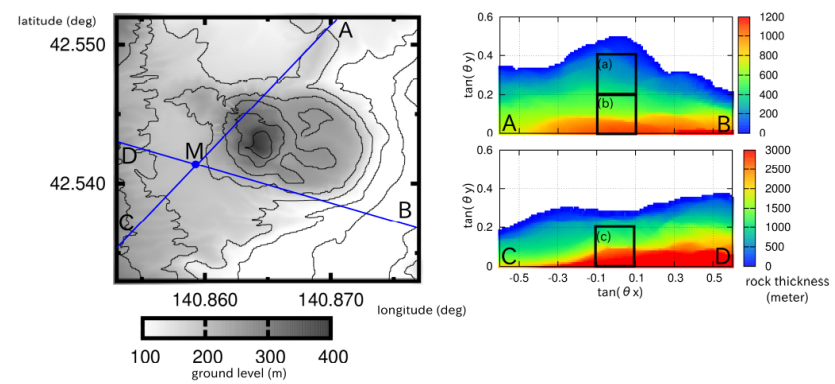

Fig. 4. Topological map of the Showa-Shinzan lava dome, Usu, Japan. "M" shows the position of our emulsion detectors. The observation ranges of the muon detector are given by $\angle \mathrm{AMB}$ (forward) and $\angle \mathrm{CMD}$ (backward). The thicknesses of the material along the radial directions are represented in the two color-contour maps to the right. The squares (a), (b) and (c) indicate three angular domains for which the particle fluxes are estimated.

\section{Analysis}

\subsection{Data acquisition and track reconstruction}

After the films were developed, the tracks recorded in the emulsion films were read using the European Scanning System (Arrabito et al., 2006). The readout system outputs the position, direction and linear density of the identified silver grains. Film-to-film alignment and track reconstruction were performed using the FEDRA (Framework for Emulsion Data Reconstruction and Analysis) framework (Tioukov et al., 2006). We then applied several cuts to the reconstructed tracks.

\subsection{Track selection}

\subsubsection{Grain density cut}

Minimum ionizing particles (MIPs) are selected by removing tracks with grain densities higher than a certain threshold. The grain density is the number of silver grains per unit length along the track and is almost proportional to the ionizing power of the incident particles. This methodology has been established by several precedent studies. For instance, Toshito et al. (2004) succeeded in discriminating $1.2 \mathrm{GeV} \mathrm{c}^{-1}$ pions (MIPs) from $1.2 \mathrm{GeV} \mathrm{c}^{-1}$ protons (nonMIPs) in an accelerator beam.

\subsection{2 $\chi^{2}$-cut}

In order to select tracks with straight trajectories in the detector, we apply a chi-square cut based on the deflection angle. $\chi^{2}$ is defined as

$\chi^{2}=\sum\left(\frac{\Delta \theta_{\mathrm{R}, i}^{2}}{\sigma_{\mathrm{R}}^{2}}+\frac{\Delta \theta_{\mathrm{T}, i}^{2}}{\sigma_{\mathrm{T}}^{2}}\right)$, 


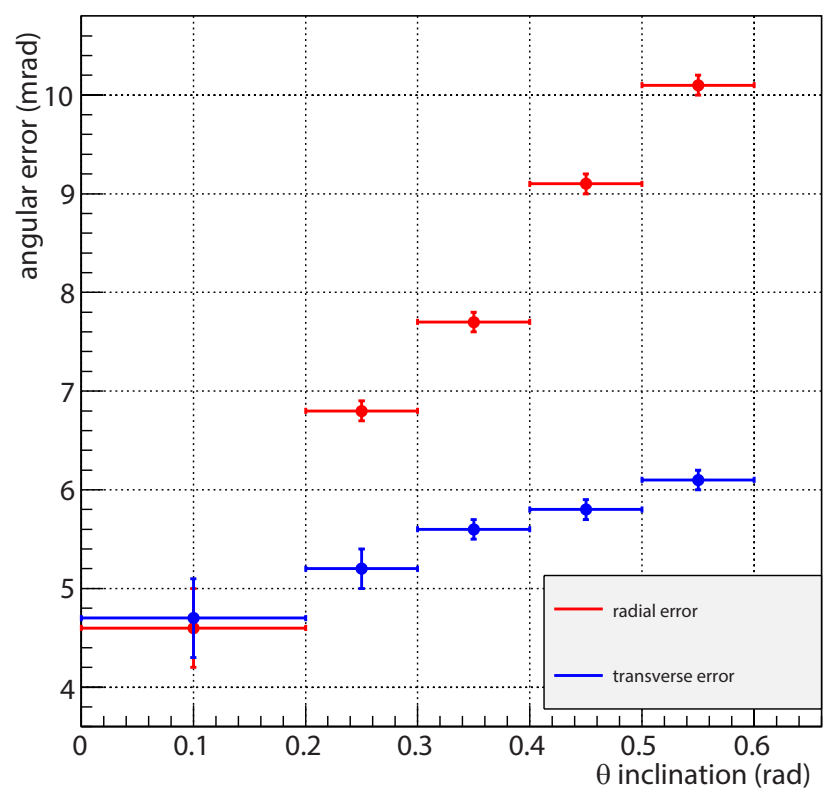

Fig. 5. Angular resolutions in the radial (red) and transverse (blue) coordinates. The horizontal axis represents the angle between a track and the normal direction of the detection plane (inclination: $\theta)$.

where $\Delta \theta_{\mathrm{R}, i}$ and $\Delta \theta_{\mathrm{T}, i}$ are the deflection angles of tracks between the $i$ th and $(i+1)$ th films in the radial and transverse coordinates, and $\sigma_{\mathrm{R}, \mathrm{T}}$ are angular measurement errors, as shown in Fig. 5. In the RT (Radial and Transverse) coordinate system, while the radial error depends severely on $\theta$ with an increase rate of $10 \mathrm{mrad} \mathrm{rad}^{-1}$, the transverse error is less dependent on $\theta\left(2 \mathrm{mrad} \mathrm{rad}^{-1}\right)$ (De Serio et al., 2003). The resultant $\chi^{2}$ values are shown in Fig. 6. We selected tracks with $p$ values $\geq 0.01(1 \%)$. Due to the finite angular resolutions of the microscopes, we regard the tracks in the tail ( $p$ values $<1 \%$ ) as noise signals, although some part of these tracks should be true muon signals.

\subsubsection{Number of hits cut}

We eliminate chance coincidences of tracks recorded during transportation using a cut based on the number of hits $(n)$ among the total number of films $(N)$. Figure 7 shows $n$ distributions for selected almost-perpendicular tracks in the quartet detector $\left(N=4,0<\theta^{\mathrm{Q}}<0.2\right)$ and the ECC detector $(N=20,0<\theta<0.4)$. Along with the number of hits distribution, we estimate the probability that the tracks recorded during transportation are mistakenly identified as signals (shaded histograms) by giving a misalignment of $500 \mu \mathrm{m}$ between adjacent films. In order to exclude these fake signals, we set the threshold as $n \geq n_{\text {cut }}^{\mathrm{Q}}=3$ for the quartet detector, and $n \geq n_{\text {cut }}^{\mathrm{ECC}}=5$ for the ECC detector. In addition to this selection, we required the tracks in the ECC detector to pass through at least five lead plates.
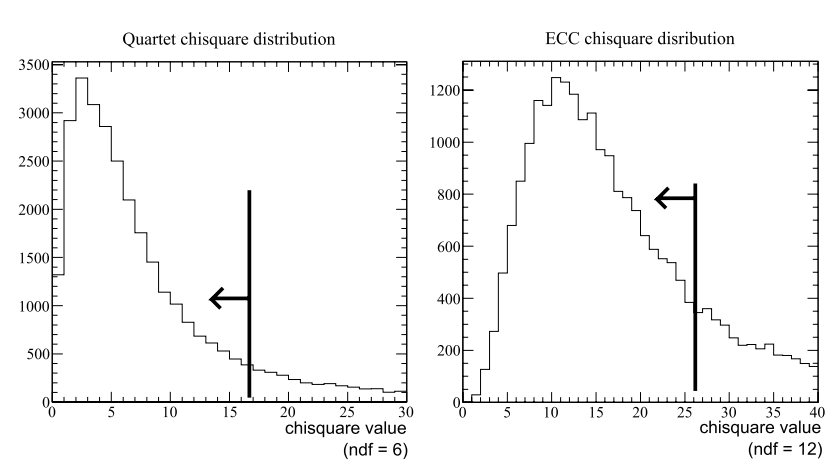

Fig. 6. Resultant distributions of $\chi^{2}$ values for the quartet detector (left: ndf $=6$; ndf - number of degrees of freedom) and ECC detector (right: $n d f=12$ ). The solid vertical line represents the upper bound we used based on the chi-square criterion ( $p$ value $>1 \%$ ).

\subsection{Efficiency study}

\subsubsection{Film efficiency}

The film efficiency is defined as the probability of detecting a track in a film when a particle passes through it. Given the efficiency values of the quartet films, $\epsilon_{1}, \epsilon_{2}, \epsilon_{3}$, and $\epsilon_{4}$, the probability that tracks are found in at least $n_{\text {cut }}^{\mathrm{Q}}=3$ of the total $N=4$ films can be expressed as

$$
\begin{aligned}
\epsilon_{\mathrm{tot}}^{\mathrm{Q}} & =\epsilon_{1} \epsilon_{2} \epsilon_{3} \epsilon_{4}+\left(1-\epsilon_{1}\right) \epsilon_{2} \epsilon_{3} \epsilon_{4}+\epsilon_{1}\left(1-\epsilon_{2}\right) \epsilon_{3} \epsilon_{4} \\
& +\epsilon_{1} \epsilon_{2}\left(1-\epsilon_{3}\right) \epsilon_{4}+\epsilon_{1} \epsilon_{2} \epsilon_{3}\left(1-\epsilon_{4}\right) .
\end{aligned}
$$

The film efficiency values are estimated for several inclination ranges from the ratio of triplet-hit signals and quartet-hit signals:

$$
\epsilon_{i}\left[\theta_{\text {min }}^{\mathrm{Q}}: \theta_{\max }^{\mathrm{Q}}\right]=\frac{N_{4}\left[\theta_{\text {min }}^{\mathrm{Q}}: \theta_{\text {max }}^{\mathrm{Q}}\right]}{N_{3, i}\left[\theta_{\text {min }}^{\mathrm{Q}}: \theta_{\max }^{\mathrm{Q}}\right]+N_{4}\left[\theta_{\text {min }}^{\mathrm{Q}}: \theta_{\max }^{\mathrm{Q}}\right]},
$$

where $N_{4}\left[\theta_{\min }^{\mathrm{Q}}: \theta_{\max }^{\mathrm{Q}}\right]$ is the number of quartet-hit tracks with inclination within $\theta_{\min }^{\mathrm{Q}} \leq \theta^{\mathrm{Q}}<\theta_{\max }^{\mathrm{Q}}$, and $N_{3, i}$ is the number of tracks found in three films and not found in the $i$ th film. As shown in Fig. 8a, the total efficiency $\epsilon_{\text {tot }}^{\mathrm{Q}}$ depends strongly on $\theta$.

For the ECC detector, the efficiency of one film is estimated by fitting the $n$ distribution with a model function. We assume the single film efficiency $\epsilon$ is a random variable following the beta distribution, beta $(\epsilon, a, b)$, with mean and variance given by $a /(a+b)$ and $a b /(a+b)^{2} /(a+b+1)$. The expected $n$ distribution can then be expressed as a betabinomial function, a compound of beta and binomial distributions:

$f(n \mid a, b)=\frac{N !}{(N-n) ! n !} \frac{B(n+a, N-n+b)}{B(a, b)}$,

where $B(\cdots)$ denotes the beta function. The model parameters $(a, b)$ are determined by fitting the observed $n$-hit 
(a).

Quartet detector

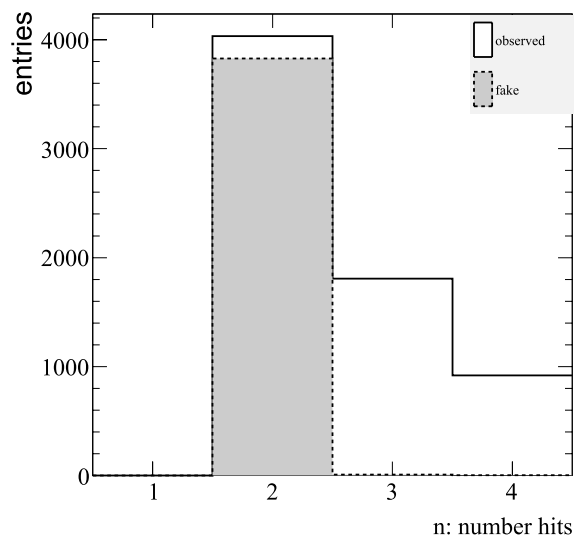

(b).

ECC detector

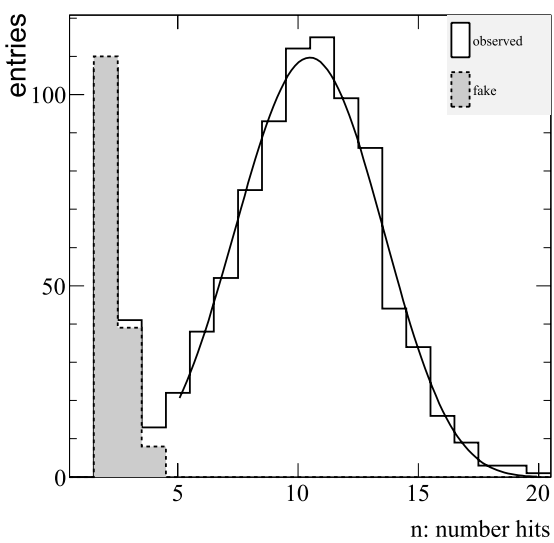

Fig. 7. (a) Number-of-hits distribution among the four films in the quartet detector for tracks with inclination $0.2 \leq \theta^{\mathrm{Q}} \leq 0.262$. The shaded histogram shows the probability that the tracks recorded during transportation are linearly aligned by chance and mistakenly identified as signals (fake signals). (b) Number-of-hits distribution among the 20 films in the ECC detector for tracks with inclination $0.2 \leq \theta \leq 0.317$, fitted with a beta-binomial distribution.
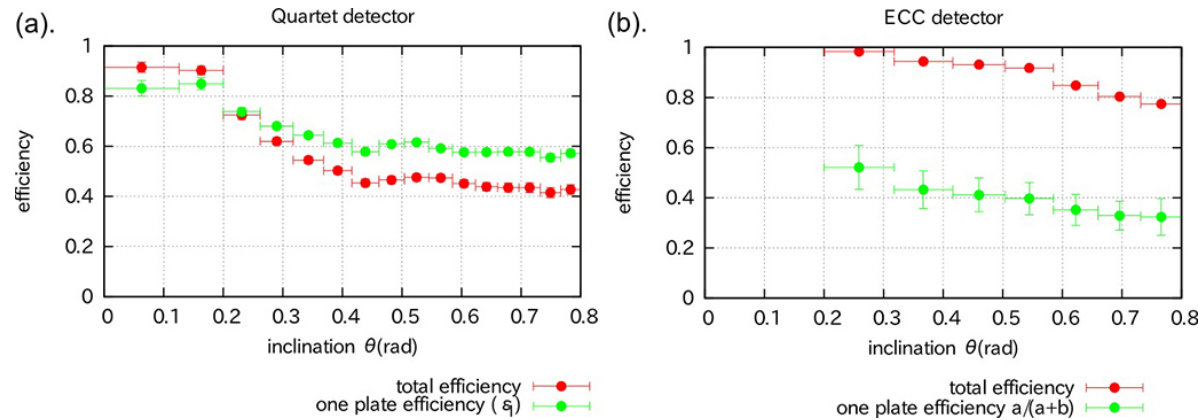

Fig. 8. Efficiency of signal detection as a function of track inclination $\theta$ for the quartet detector (a) and ECC detector (b). The green circles show the efficiency for a single film and the red circles show the total efficiency values $\epsilon$,ECC .

distribution with Eq. (6). We use the MINUIT package for this fitting. The total efficiency then becomes

$$
\begin{gathered}
\epsilon_{\mathrm{tot}}^{\mathrm{ECC}}\left[\theta_{\min }: \theta_{\max }\right]=\sum_{n=5}^{20} f\left(n \mid a\left[\theta_{\min }: \theta_{\max }\right],\right. \\
\left.b\left[\theta_{\min }: \theta_{\max }\right]\right) .
\end{gathered}
$$

We show the film/total efficiency values of the ECC as a function of $\theta$ in Fig. 8b. Although the single-film efficiency values are generally lower than the quartet values, the total efficiency is considerably higher than the quartet detector and is almost flat for $\theta<0.6$. This stable, high efficiency is achieved due to the highly redundant films of the ECC detector.

\subsubsection{Momentum thresholds}

The surviving rate of the $\chi^{2}$-cut is estimated for a variety of momenta by a Monte Carlo simulation. Using random number generators, we generated deflection angles $\Delta \theta_{\mathrm{R}, \mathrm{T}}$ for each gap in the detector and computed $\chi_{\mathrm{MC}}^{2}$ from Eq. (3). The deflection angles were given as a combination of observational angular error and scattering angle in Eq. (1):

$\Delta \theta_{\mathrm{R}, \mathrm{T}}=\zeta \sigma_{\mathrm{R}, \mathrm{T}}+\eta \sigma_{\mathrm{scat}}$,

where $\zeta$ and $\eta$ are normal random variables with a mean of zero and a variance of one. The material and gap distance are taken into account in the $t / X_{0}$ term in Eq. (1). Out of 10000 tries, the percentage where the resultant $\chi_{\mathrm{MC}}^{2}$ meets the $\chi^{2}$ criterion ( $p$ value $>1 \%$ ) is taken as the surviving rate at a given momentum $s(p)$. Figure 9 shows the surviving probability as a function of momentum for the quartet and ECC detectors. While $s(p)$ of the quartet detector approaches $90 \%$ at $0.2 \mathrm{GeV} \mathrm{c}^{-1}$, the ECC detector rejects particles of 


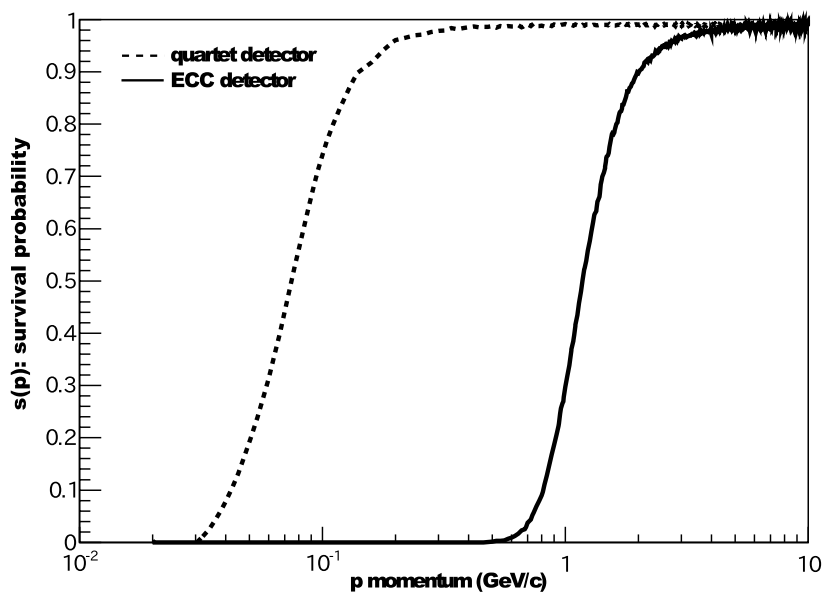

Fig. 9. Momentum dependence of the efficiency for the ECC detector (solid line) and quartet detector (dashed line).

this momentum. The ECC detector is sensitive to particles with momentum above $2.0 \mathrm{GeV} \mathrm{c}^{-1}$. Thus, we can say that the momentum thresholds of the quartet and ECC detectors are 0.2 , and $2.0 \mathrm{GeV} \mathrm{c}^{-1}$, respectively. It should be also noted that ambient beta and gamma rays (submegaelectronvolt $\sim \mathrm{a}$ few megaelectronvolt) are completely excluded with the aid of this momentum cut.

\subsection{Particle flux calculation}

A particle flux from a solid angle spanned by $D\left[\tan \theta_{x, \min }\right.$ : $\left.\tan \theta_{x, \max }, \tan \theta_{y, \min }: \tan \theta_{y, \max }\right]$ is derived from the number of selected tracks and the total efficiency values:

$F^{\mathrm{Q}, \mathrm{ECC}}=\frac{1}{\int_{D} S \cos \theta T \mathrm{~d} \Omega}\left(\sum_{i} \frac{N\left[\theta_{i, \min }: \theta_{i, \max }\right]}{\epsilon_{\mathrm{tot}}\left[\theta_{i, \min }: \theta_{i, \max }\right]}\right)$,

where $N\left[\theta_{i, \min }: \theta_{i, \max }\right]$ denotes the number of selected tracks in the $i$ th inclination range, $S$ the area of the film in the analysis, $\Omega$ the solid angle, and $T$ the exposure time.

\subsection{Simulated muon flux}

For comparison with the observed particle fluxes, we calculate the expected values of the muon flux for a variety of values of topographic density $\rho_{\text {sim }}$. The calculation was performed by subdividing the view into small sections and summing the integrated theoretical muon energy spectrum above the cutoff energies:

$$
\begin{aligned}
F^{\mathrm{SIM}} & =\frac{1}{\sum_{x, y} S \cos \theta_{x, y} T \Delta \Omega_{x, y}} \\
& \left\{\sum_{x, y} S \cos \theta_{x, y} T \Delta \Omega_{x, y} \int_{E_{\text {cut }}\left(X_{x, y}\right)}^{\infty} f_{\mu}\left(\vartheta_{x, y}, E\right) \mathrm{d} E\right\} .
\end{aligned}
$$

Here, $\Delta \Omega_{x, y}$ and $\vartheta_{x, y}$ are the solid angle and the zenith angle for the subdivided view with intervals of
$\left[\Delta \tan \theta_{x}, \Delta \tan \theta_{y}\right]=[0.01,0.01]$. The energy-cutoff $E_{\text {cut }}$ value for a given density length is taken from the muon-range table (Groom et al., 2001). The density length of the rock along the radial direction for the subdivided view was given by $X_{x, y}=\rho_{\text {sim }} \times$ (thickness of rock).

\subsection{Results}

We summarize the results of our experiment in Table 1. We estimate the particle fluxes $\left(F^{\mathrm{Q}, \mathrm{ECC}}\right)$ for three angular domains, in which the rock thickness along the radial directions are (a) 70-469m, (b) 432-1100m, and (c) $907-3879 \mathrm{~m}$, as shown in Fig. 4. Using the observed fluxes, we estimate the average density of rock in each view by comparing them with the simulated flux (Fig. 10). The quartet detector yields an average density of $1.18_{-0.04}^{+0.04}, 0.76_{-0.05}^{+0.05}$, and $0.23_{-0.02}^{+0.02} \mathrm{~g} \mathrm{~cm}^{-3}$ for domains (a), (b), and (c), respectively. The ECC detector yields $2.33_{-0.10}^{+0.12}$ and $2.00_{-0.17}^{+0.23} \mathrm{~g} \mathrm{~cm}^{-3}$ for domains (a) and (b). For domain (c), we could not determine the average density due to the lack of statistics.

For each domain (a), (b), and (c), the particle-flux estimated from the quartet detector is much higher than that from the ECC detector. Considering the different momentum thresholds for the two detectors, this difference in flux arises from the low-momenta particles in $0.2 \mathrm{GeV} \mathrm{c}^{-1}<p<$ $2 \mathrm{GeV} \mathrm{c}^{-1}$. While the density values determined from the ECC detector are consistent with the density of typical volcanic rocks, the density values determined from the quartet detector are significantly lower. This fact suggests that while the ECC detects only the signal muons, the quartet detector is affected by contamination from other low-momentum particles.

\section{Discussion and conclusion}

This study confirms that the source of background noise in muon radiography is charged particles with momenta < $2 \mathrm{GeV} \mathrm{c}^{-1}$. It also demonstrates that a momentum-discerning detector, like an ECC detector, can reduce the contamination from background noise to negligible levels.

We have also compared our conclusion with some earlier studies of the background noise particles in muon radiography. Tanaka et al. (2011a) discussed false muon tracks that arise when several particles accidentally hit several counter planes at the same time within the time resolution of the electronic muon detector. This type of noise is negligible in emulsion detectors because emulsion detectors have high position and direction resolution, and the frequency of random coincidence of the tracks is lowered to negligible levels as a result of the number of hits cut. Lesparre et al. (2012) discussed the contamination from electromagnetic components of airshower particles. They inserted $24 \mathrm{~mm}$-thick iron plates between the counter planes to stop $e^{+} / e^{-}$particles with kinetic 

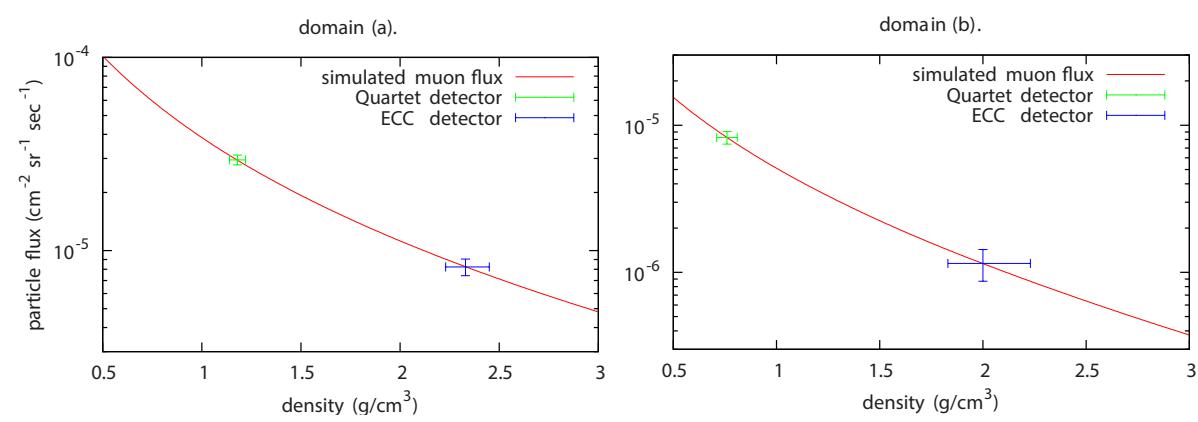

Fig. 10. Simulated muon flux against assumed density for two angular domains, (a) left and (b) right. The particle fluxes observed in the quartet and ECC detectors are shown by green and blue vertical error bars $(1 \sigma)$. The estimated density values are shown by the horizontal error bars.

Table 1. The number of the selected tracks for the three angular domains (a), (b), and (c) for the quartet detector (top) and ECC detector (bottom). The last rows show the particle fluxes with efficiency compensation using $\epsilon_{\text {tot }}$ values for each inclination range.

\begin{tabular}{|c|c|c|c|c|}
\hline \multicolumn{5}{|c|}{ Quartet detector: $S=27 \mathrm{~cm}^{2}, T=1.45 \times 10^{7} \mathrm{~s}$} \\
\hline inclination range: $\theta^{\mathrm{Q}}$ & domain (a) & domain (b) & domain (c) & efficiency $(\%): \epsilon_{\text {tot }}^{\mathrm{Q}}$ \\
\hline $0-0.126$ & 151 & 71 & - & $91.5 \pm 2.0$ \\
\hline $0.126-0.200$ & 168 & 34 & - & $90.2 \pm 1.8$ \\
\hline $0.200-0.262$ & 1 & 3 & 27 & $72.4 \pm 1.7$ \\
\hline $0.262-0.317$ & - & - & 20 & $62.0 \pm 1.4$ \\
\hline $0.317-0.368$ & - & - & 28 & $54.5 \pm 1.4$ \\
\hline $0.368-0.416$ & - & - & 27 & $50.3 \pm 1.5$ \\
\hline $0.416-0.461$ & - & - & 2 & $45.4 \pm 1.5$ \\
\hline total statistics & 320 & 108 & 104 & \\
\hline flux: $F \mathrm{Q}_{\left(\mathrm{cm}^{-2} \mathrm{sr}^{-1} \mathrm{~s}^{-1}\right)}$ & $(2.97 \pm 0.17) \times 10^{-5}$ & $(8.25 \pm 0.80) \times 10^{-6}$ & $(1.19 \pm 0.12) \times 10^{-5}$ & \\
\hline estimated density $\left(\mathrm{g} \mathrm{cm}^{-3}\right)$ & $1.18_{-0.04}^{+0.04}$ & $0.76_{-0.05}^{+0.05}$ & $0.23_{-0.02}^{+0.02}$ & \\
\hline
\end{tabular}

\begin{tabular}{lrrrr}
\hline & ECC detector: $S=27 \mathrm{~cm}^{2}, T=1.45 \times 10^{7} \mathrm{~s}$ & & \\
\hline inclination range: $\theta$ & domain (a) & domain (b) & domain (c) & efficiency $(\%): \epsilon_{\text {tot }}^{\text {ECC }}$ \\
\hline $0-0.200$ & - & 15 & 2 & $>98.4$ \\
$0.200-0.317$ & 27 & 2 & - & -0.02 \\
$0.317-0.416$ & 75 & 17 & 2 & $94.4 \pm 0.3$ \\
total statistics & 102 & $2.00_{-0.17}^{+0.23}$ & too low statistics \\
flux: $F^{\mathrm{ECC}}\left(\mathrm{cm}^{-2} \mathrm{sr}^{-1} \mathrm{~s}^{-1}\right)$ & $(8.23 \pm 0.81) \times 10^{-6}$ & $(1.15 \pm 0.28) \times 10^{-6}$ &
\end{tabular}

energies $<108.3 \mathrm{MeV}$. In our study, the quartet detector also excludes particles in this energy range; however, we still see an excess of particle flux. This difference should be further studied with consideration of detector dependence problems. Jourde et al. (2013) found the upward-going particles that come from the backward free sky with a dedicated time-offlight analysis of scintillation type detectors. This type of particles definitely cause noise signals also for emulsion detectors. However, we did not find an excess of the particle flux in the backward direction for the ECC detector. This is probably because there is a significant amount of rock in the backward direction for our detectors (Mt. Usu).
It has not yet been determined whether low-momentum noise particles consist of electromagnetic components or scattered muons, since our ECC detector does not identify the type of particle because the thickness of the absorbing material in the detector is not sufficient for electromagnetic calorimetry. While the muon energy spectrum drops below $1 \mathrm{GeV}$ due to decay, the electron energy spectrum does not drop down to its critical energy in the air $(81 \mathrm{MeV})$. This implies that most of cosmic electrons at sea level have a low energy of around $81 \mathrm{MeV}$ and that they have typically large scattering angles in the atmosphere. Thus these wellscattered electrons hit the detector randomly. It should be noted that these electrons come from the direction of the 
mountain, which resulted in the excess of the flux for the quartet detector and deteriorated density estimation of the mountain.

Our result does not exclude the possibility that the cosmic muons, scattered in the topographic material, should be the source of background noise. Figure 1b shows that most of the well-scattered muons have momenta of lower than $2 \mathrm{GeV} \mathrm{c}^{-1}$; and they were properly excluded in our ECC detector. However, a very small amount of middle-momentum muons (blue: $2 \mathrm{GeVc}^{-1}<p<10 \mathrm{GeVc}^{-1}$ ) have a scattering angle as large as $100 \mathrm{mrad}$. The remaining problem is how many of these muons can reach and hit the detector. This should be further studied through a whole Monte Carlo simulation considering particle scattering in the atmosphere and topography, and particle deflection in the geomagnetic field of the Earth. There are some pioneering works of such large-scale computation. For instance, Honda et al. (2004) performed atmospheric neutrino-flux calculations in a threedimensional space with an IGRF (International Geomagnetic Reference Field) geomagnetic model for neutrino oscillation experiments. Béné et al. (2013) attempted to quantify the amount of accidental coincidences produced in the detector by air-shower induced particles.

We have demonstrated that the high position/angular resolutions of emulsion detectors enable high-S / N (signal-tonoise) muography observations. The remaining task is to fasten the read-out speed of microscopes for the future application with larger detectors. The upgrading of the read-out system is under development by several groups (e.g., Bozza et al., 2013; Yoshimoto, 2013).

Acknowledgements. The authors wish to thank M. Nakamura and his colleagues for their support in preparing and developing the emulsion films. Installation of the emulsion detectors was supported by $\mathrm{H}$. Oshima and T. Maekawa from Hokkaido University. Beautification Escort Staff (BES) at Shikotsu-Toya National Park provided us with a site for our observation. We are grateful to C. Bozza for his continuing support in the use of the European Scanning System. We acknowledge with gratitude the tremendous support given us in the use of the FEDRA framework by V. Tioukov. We thank A. Taketa, H. K. M. Tanaka and S. Okubo for their encouragement and valuable suggestions. Valuable questions and comments from four anonymous referees were of enormous help to improve the manuscript. This paper greatly benefited from proofreading/editing assistance provided by the GCOE program From the Earth to Earths. During this work, one of the authors (R. Nishiyama) was supported by a JSPS fellowship (24-9317).

Edited by: W. Schmidt

\section{References}

Agostinelli, S., Allison, J., Amako, K., Apostolakis, J., Araujo, H., Arce, P., Asai, M., Axen, D., Banerjee, S., Barrand, G., Behner, F., Bellagamba , L., Boudreau, J., Broglia, L., Brunengo, A.,
Burkhardt, H., Chauvie, S., Chuma, J., Chytracek, R., Cooperman, G., Cosmo, G., Degtyare, S., Giani, S., Giannitrapani, R., Gibin, D., Gómez Cadenas, J. J., González, I., Gracia Abril, G., Greeniaus, G., Greiner, W., Grichine, V., Grossheim, A., Guatelli, S., Gumplinger, P., Hamatsu, R., Hashimoto, K., Hasui, H., Heikkinen, A., Howard, A., Ivanchenko, V., Johnson, A., Jones, F. W., Kallenbach, J., Kanaya, N., Kawabata, M., Kawabata, Y., Kawaguti, M., Kelner, S., Kent, P., Kimura, A., Kodama, T., Kokoulin, R., Kossov, M., Kurashige, H., Lamanna, E., Lampén, T., Lara, V., Lefebure, V., Le, E., Minamimoto, K., Mora de Freitas, P., Morita, Y., Murakami, K., Nagamatu, M., Nartallo, R., Nieminen, P., Nishimura, T., Ohtsubo, K., Okamura, M., O’Neale, S., Oohata , Y., Paech, K., Perl, J., Pfeiffer, A., Pia, M. G., Ranjard, F., Rybin, A., Sadilov, S., Di Salvo, E., Santin, G., Sasaki, T., Savvas, N., Sawada, Y., Scherer, S., Sei, S., Sirotenko, V., Smith, D., Starkov, N., Stoecker, H., Sulkimo, J., Takahata, M., Tanaka, S., Tcherniaev, E., Safai Tehrani, E., Tropeano, M., Truscott, P., Uno, H., Urban, L., Urban, P., Verderi, M., Walkden, A., Wander, W., Weber, H., Wellisch, J. P., Wenaus, T., Williams, D. C., Wright, D., Yamada, T., Yoshida, H., and Zschiesche, D.: GEANT4 - a simulation toolkit, Nucl. Instrum. Meth. A, 506, 250-303, 2003.

Allkofer, O., Bella, G., Dau, W., Jokisch, H., Klemke, G., Oren, Y., and Uhr, R.: Cosmic ray muon spectra at sea-level up to $10 \mathrm{TeV}$, Nucl. Phys. B, 259, 1-18, 1985.

Anastasio, A., Ambrosino, F., Basta, D., Bonechi, L., Brianzi, M., Bross, A., Callier, S., Caputo, A., Ciaranfi, R., Cimmino, L., D’Alessandro, R., D’Auria, L., Taille, C., Energico, S., Garufi, F., Giudicepietro, F., Lauria A., Macedonio, G., Martini, M., Masone, V., Mattone, C., Montesi, M. C., Noli, P., Orazi, M., Passeggio, G., Peluso, R., Pla-Dalmau, A., Raux, L., Rubinov, P., Saracino, G., Scarlini, E., Scarpato, G., Sekhniaidze, G., Starodubtsev, O., Strolin, P., Taketa, A., Tanaka, H. K. M. and Vanzanella, A.: The MU-RAY detector for muon radiography of volcanoes, Nucl. Instrum. Meth. A, 732, 423-426, 2013.

Arrabito, L., Barbuto, E., Bozza, C., Buontempo, S., Consiglio, L., Coppola, D., Cozzi, M., Damet, J., D’Ambrosio, N., De Lellis, G., De Serio, M., Di Capua, F., Di Ferdinando, D., Di Marco, D., Esposito, L. S., Giacomelli, G., Grella, G., Hauger, M., Juget, F., Kreslo, I., Giorgini, M., Ieva, M., Laktineh, I., Manai, K., Mandrioli, G., Marotta, A., Manzoor, S., Migliozzi, P., Monacelli, P., Muciaccia, M. T., Pastore, A., Patrizii, L., Pistillo, C., Pozzato, M., Royole-Degieux, P., Romano, G., Rosa, G., Savvinov, N., Schembri, A., Scotto Lavina, L., Simone, S., Sioli, M., Sirignan, C., Sirri, G., Sorrentino, G., Strolin, P., Tioukov, V., and Waelchli, T.: Hardware performance of a scanning system for high speed analysis of nuclear emulsions, Nucl. Instrum. Meth. A, 568, 578587, 2006.

Barnaföldi, G. G., Hamar, G., Melegh, H. G., Oláh, L., Surányi, G., and Varga, D.: Portable cosmic muon telescope for environmental applications, Nucl. Instrum. Meth. A, 689, 60-69, 2012

Béné, S., Boivin, P., Busato, E., Cârloganu, C., Combaret, C., Dupieux, P., Fehr, F., Gay, P., Labazuy, P., Laktineh, I., Lénat, J.F., Miallier, D., Mirabito, L., Niess, V., Portal, A., and Vulpescu, B.: Air shower simulation for background estimation in muon tomography of volcanoes, Geosci. Instrum. Method. Data Syst., 2, 11-15, doi:10.5194/gi-2-11-2013, 2013.

Beringer, J., Arguin, J.-F., Barnett, R. M., Copic, K., Dahl, O., Groom, D. E., Lin, C.-J., Lys, J., Murayama, H., Wohl, C. G., 
Yao, W.-M., Zyla, P. A., Amsler, C., Antonelli, M., Asner, D. M., Baer, H., Band, H. R., Basaglia, T., Bauer, C. W., Beatty, J. J., Belousov, V. I., Bergren, E., Bernardi, G., Bertl, W., Bethke, S., Bichsel, H., Biebel, O., Blucher, E., Blusk, S., Brooijmans, G., Buchmueller, O., Cahn, R. N., Carena, M., Ceccucci, A., Chakraborty, D., Chen, M.-C., Chivukula, R. S., Cowan, G., D’Ambrosio, G., Damour, T., de Florian, D., de Gouvêa, A., DeGrand, T., de Jong, P., Dissertori, G., Dobrescu, B., Doser, M., Drees, M., Edwards, D. A., Eidelman, S., Erler, J., Ezhela, V. V., Fetscher, W., Fields, B. D., Foster, B., Gaisser, T. K., Garren, L., Gerber, H.-J., Gerbier, G., Gherghetta, T., Golwala, S., Goodman, M., Grab, C., Gritsan, A. V., Grivaz, J.-F., Grünewald, M., Gurtu, A., Gutsche, T., Haber, H. E., Hagiwara, K., Hagmann, C., Hanhart, C., Hashimoto, S., Hayes, K. G., Heffner, M., Heltsley, B., Hernández-Rey, J. J., Hikasa, K., Höcker, A., Holder, J., Holtkamp, A., Huston, J., Jackson, J. D., Johnson, K. F., Junk, T., Karlen, D., Kirkby, D., Klein, S. R., Klempt, E., Kowalewski, R. V., Krauss, F., Kreps, M., Krusche, B., Kuyanov, Yu. V., Kwon, Y., Lahav, O., Laiho, J., Langacker, P., Liddle, A., Ligeti, Z., Liss, T. M., Littenberg, L., Lugovsky, K. S., Lugovsky, S. B., Mannel, T., Manohar, A. V., Marciano, W. J., Martin, A. D., Masoni, A., Matthews, J., Milstead, D., Miquel, R., Mönig, K., Moortgat, F., Nakamura, K., Narain, M., Nason, P., Navas, S., Neubert, M., Nevski, P., Nir, Y., Olive, K. A., Pape, L., Parsons, J., Patrignani, C., Peacock, J. A., Petcov, S. T., Piepke, A., Pomarol, A., Punzi, G., Quadt, A., Raby, S., Raffelt, G., Ratcliff, B. N., Richardson, P., Roesler, S., Rolli, S., Romaniouk, A., Rosenberg, L. J., Rosner, J. L., Sachrajda, C. T., Sakai, Y., Salam, G. P., Sarkar, S., Sauli, F., Schneider, O., Scholberg, K., Scott, D., Seligman, W. G., Shaevitz, M. H., Sharpe, S. R., Silari, M., Sjöstrand, T., Skands, P., Smith, J. G., Smoot, G. F., Spanier, S., Spieler, H., Stahl, A., Stanev, T., Stone, S. L., Sumiyoshi, T., Syphers, M. J., Takahashi, F., Tanabashi, M., Terning, J., Titov, M., Tkachenko, N. P., Törnqvist, N. A., Tovey, D., Valencia, G., van Bibber, K., Venanzoni, G., Vincter, M. G., Vogel, P., Vogt, A., Walkowiak, W., Walter, C. W., Ward, D. R., Watari, T., Weiglein, G., Weinberg, E. J., Wiencke, L. R., Wolfenstein, L., Womersley, J., Woody, C. L., Workman, R. L., Yamamoto, A., Zeller, G. P., Zenin, O. V., Zhang, J., Zhu, R.-Y., Harper, G., Lugovsky, V. S., and Schaffner, P.: Review of particle physics, Phys. Rev. D, 86, 010001, doi:10.1103/PhysRevD.86.010001, 2012.

Bozza, C., Consiglio, L., D’Ambrosio, N., De Lellis, G., De Sio, C., Di Marco, N., Kose, U., Medinaceli, E., Miyamoto, S., Nishiyama, R., Pupilli, F., Stellacci, S. M., Sirignano, C., Tanaka, H. K. M., and Tioukov, V.: Muon radiography by nuclear emulsions: data acquisition and processing, IAVCEI 2013 Scientific Assembly, Kagoshima, Japan, 20-24 July, 2013, 1P2_2D-O27, 2013.

Cârloganu, C., Niess, V., Béné, S., Busato, E., Dupieux, P., Fehr, F., Gay, P., Miallier, D., Vulpescu, B., Boivin, P., Combaret, C., Labazuy, P., Laktineh, I., Lénat, J.-F., Mirabito, L., and Portal, A.: Towards a muon radiography of the Puy de Dôme, Geosci. Instrum. Method. Data Syst., 2, 55-60, doi:10.5194/gi-2-55-2013, 2013.

De Serio, M., Ieva, M., Simone, S., Giorgini, M., Sioli, M., Sirri, G., Buontempo, S., D’Ambrosio, N., De Lellis, G., De Rosa, G., Barbuto, E., Bozza, C., and Sirignano, C.: Momentum measurement by the angular method in the Emulsion Cloud Chamber, Nucl. Instrum. Meth. A, 512, 539-545, 2003.
Golden, R., Stephens, S., Stochaj, S., Webber, W., Brunetti, M., Codino, A., Grimani, C., Menichelli, M., Salvatori, I., De Pascale, M. P., Morselli, A., Picozza, P., Basini, G., Bongiorno, F., Brancaccio, F. M., Ricci, M., Ormes, J. F., Streitmatter, R. E., Papini, P., Piccardi, S., and Spillantini, P.: Measurement of the energy spectra of cosmic ray electron component and protons at ground level, J. Geophys. Res., 100, 23515-23523, 1995.

Groom, D. E., Mokhov, N. V., and Striganov, S. I.: Muon Stopping Power and Range Tables $10 \mathrm{MeV}-100 \mathrm{TeV}$, Atom. Data Nucl Data Tab., 78, 183-356, 2001.

Honda, M., Kajita, T., Kasahara, K., and Midorikawa, S.: New calculation of the atmospheric neutrino flux in a three-dimensional scheme, Phys. Rev. D, 70, 043008 , doi:10.1103/PhysRevD.70.043008, 2004.

Japan Meteorological Agency: http://www.jma.go.jp/jma/indexe. html (last access: 20 February 2014).

Jourde, K., Gibert, D., Marteau, J., De Bermond D'ars, J., Gardien, S., Girerd, C., Ianigro, J. C., and Carbone, D.: Experimental detection of upward going cosmic particles and consequences for correction of density radiography of volcanoes, Geophys. Res. Lett., 40, 6334-6339, doi:10.1002/2013GL058357, 2013.

Lesparre, N., Gibert, D., Marteau, J., Komorowski, J.-C., Nicollin, F., and Coutant, O.: Density muon radiography of La Soufriere of Guadeloupe volcano: comparison with geological, electrical resistivity and gravity data, Geophys. J. Int., 190, 1008-1019, 2012.

Lynch, G. R. and Dahl, O. I.: Approximations to multiple Coulomb scattering, Nucl. Instrum. Meth. B, 58, 6-10, 1991.

Matsuno, S., Kajino, F., Kawashima, Y., Kitamura, T., Mitsui, K., Muraki, Y., Ohashi, Y., Okada, A., Suda, T., Minorikawa, Y., Kobayakawa, K., Kamiya, Y., Nakamura, I., and Takahashi, T.: Cosmic-ray muon spectrum up to $20 \mathrm{TeV}$ at $89^{\circ}$ zenith angle, Phys. Rev. D, 29, 1-23, doi:10.1103/PhysRevD.29.1, 1984.

Morishima, K. and Nakano, T.: Development of a new automatic nuclear emulsion scanning system, S-UTS, with continuous 3-D tomographic image read-out, J. Instr., 5, P04011, doi:10.1088/1748-0221/5/04/P04011, 2010.

Nakamura, T., Ariga, A., Ban, T., Fukuda, T., Fukuda, T., Fujioka, T., Furukawa, T., Hamada, K., Hayashi, H., Hiramatsu, S., Hoshino, K., Kawada, J., Koike, N., Komatsu, M., H Matsuoka, H., Miyamoto, S., Miyanishi, K., Miyanishi, M., Morishima, K., Nada, H., Naganawa, N., Nakano, T., Narita, K., Natsume, M., Niwa, K., Nonaka, N., Park, B. D., Sato, O., Takahashi, S., Toshito, T., Uetake, T., Nakamura, M., Kuwabara, K., Nishiyama, S., Nonoyama, Y., and Kodama, K.: The OPERA film: new nuclear emulsion for large-scale, high-precision experiments, Nucl. Instrum. Meth. A, 556, 80-86, 2006.

Portal, A., Labazuy, P., Lénat, J.-F., Béné, S., Boivin, P., Busato, E., Cârloganu, C., Combaret, C., Dupieux, P., Fehr, F., Gay, P., Laktineh, I., Miallier, D., Mirabito, L., Niess, V., and Vulpescu, B.: Inner structure of the Puy de Dôme volcano: cross-comparison of geophysical models (ERT, gravimetry, muon imaging), Geosci. Instrum. Method. Data Syst., 2, 47-54, doi:10.5194/gi-2-472013, 2013.

Tanaka, H., Nakano, T., Takahashi, S., Yoshida, J., and Niwa, K.: Development of an emulsion imaging system for cosmic-ray muon radiography to explore the internal structure of a volcano, Mt. Asama, Nucl. Instrum. Meth. A, 575, 489-497, 2007. 
Tanaka, H., Uchida, T., and Tanaka, M.: Newly Developed MultiLayered Muon Detection System for the Next Generation of Muon Radiography, in: EGU General Assembly, EGU20112932, Vienna, Austria, 2011a.

Tanaka, H. K., Miyajima, H., Kusagaya, T., Taketa, A., Uchida, T., and Tanaka, M.: Cosmic muon imaging of hidden seismic fault zones: rainwater permeation into the mechanical fractured zones in Itoigawa-Shizuoka Tectonic Line, Japan, Earth Planet. Sc. Lett., 306, 156-162, 2011b.

Tioukov, V., Kreslo, I., Petukhov, Y., and Sirri, G.: The FEDRA - Framework for emulsion data reconstruction and analysis in the OPERA experiment, Nucl. Instrum. Meth. A, 559, 103-105, 2006.
Toshito, T., Ariga, A., Ban, T., Hoshino, K., Komatsu, M., Naganawa, N., Nakamura, M., Nakamura, T., Nakano, T., and Niwa, K.: $\pi / p$ separation at $1.2 \mathrm{GeV} \mathrm{c}^{-1}$ by an emulsion cloud chamber, Nucl. Instrum. Meth. A, 516, 436-439, 2004.

Yoshimoto, M.: Development of nuclear emulsion readout system HTS (Hyper Track Selector) and an application to muon tomography, Muon Neutrino Radiography workshop, Tokyo, Japan, 25-26 July, available at: http://www.eri.u-tokyo.ac.jp/ht/ MNR13/program_pdf/MNR2013_HTS_Yoshimoto.pdf (last access: 20 February 2014), 2013. 